- Orthodontics can be used to develop the peri-implant site prior to implant placement.

- Orthodontics can be used to create space for implant placement

- Dental implants may be used for absolute anchorage.

- Many types of dental implants can be used to provide anchorage.

\title{
The role of orthodontics in implant dentistry
}

\author{
T. P. Rose, ${ }^{1}$ S. Jivraj ${ }^{2}$ and W. Chee $^{3}$
}

\begin{abstract}
Orthodontic treatment of partially edentulous patients is difficult, especially if a significant number of teeth are missing. With loss of teeth, adjacent or opposing teeth usually tip, drift or over-erupt leaving spaces that are not optimal for replacement of missing teeth. Orthodontic correction of these spatial relationships will aid prosthetic replacement of the missing teeth, function, hygiene and aesthetics. Orthodontists rely on teeth to provide the anchorage to correct malocclusions. With patients with an intact dentition dental anchorage is usually adequate to facilitate tooth movement. In some partially edentulous patients however, insufficient anchorage may present to correct the malocclusion. In these patients implants can provide additional anchorage. At times, osseointegrated implants can also be used to support restorations after completion of orthodontic therapy if treatment planning is precise. The use of implants for orthodontic anchorage requires an interdisciplinary approach and precise planning to achieve optimal results.
\end{abstract}

\begin{tabular}{|c|}
\hline IMPLANTS \\
\hline 1. Rationale for dental implants \\
\hline $\begin{array}{l}\text { 2. Treatment planning of implants in } \\
\text { posterior quadrants }\end{array}$ \\
\hline $\begin{array}{l}\text { 3. Treatment planning of implants in } \\
\text { the aesthetic zone }\end{array}$ \\
\hline $\begin{array}{l}\text { 4. Surgical guidelines for dental } \\
\text { implant placement }\end{array}$ \\
\hline $\begin{array}{l}\text { 5. Immediate implant placement: } \\
\text { treatment planning and surgical steps } \\
\text { for successful outcomes }\end{array}$ \\
\hline $\begin{array}{l}\text { 6. Treatment planning of the } \\
\text { edentulous maxilla }\end{array}$ \\
\hline $\begin{array}{l}\text { 7. Treatment planning of the } \\
\text { edentulous mandible }\end{array}$ \\
\hline $\begin{array}{l}\text { 8. Impressions techniques for implant } \\
\text { dentistry }\end{array}$ \\
\hline $\begin{array}{l}\text { 9. Screw versus cemented implant } \\
\text { supported restorations }\end{array}$ \\
\hline $\begin{array}{l}\text { 10. Designing abutments for } \\
\text { cement retained implant supported } \\
\text { restorations }\end{array}$ \\
\hline 11. Connecting implants to teeth \\
\hline $\begin{array}{l}\text { 12. Transitioning a patient from teeth } \\
\text { to implants }\end{array}$ \\
\hline $\begin{array}{l}\text { 13. The role of orthodontics in } \\
\text { implant dentistry }\end{array}$ \\
\hline $\begin{array}{l}\text { 14. Interdisciplinary approach to } \\
\text { implant dentistry }\end{array}$ \\
\hline $\begin{array}{l}\text { 15. Factors that affect individual } \\
\text { tooth prognosis and choices in } \\
\text { contemporary treatment planning }\end{array}$ \\
\hline 16. Maintenance and failures \\
\hline
\end{tabular}

The incorporation of orthodontic therapy into comprehensive treatment planning is a valuable adjunct which can enhance aesthetic and functional outcomes in restorative dentistry.

When treatment planning for implant supported restorations the orthodontist can assist the restorative dentist in several ways.

\section{Orthodontics to develop the peri-implant site}

Aesthetic implant placement is driven by both a restorative and biological philosophy. Aesthetically the implant should be placed to satisfy the parameters of contour so that the restoration is pleasing. Biologically it should be placed to allow maintenance of both hard and soft tissue architecture. If the tooth to be replaced has not yet been removed, several determina-

\footnotetext{
${ }^{1 *}$ Orthodontic consultant to the Craniofacial Team, Children's Hospital of Orange County (CHOC), Orange County, California; ${ }^{2}$ Chairman, Section of Fixed Prosthodontics and Operative Dentistry, University of Southern California School of Dentistry / Private Prosthodontics Practitioner, Burbank, California; ${ }^{3}$ Ralph W. and Jean L. Bleak Professor of Restorative Dentistry, Director of Implant Dentistry at the University of Southern California School of Dentistry / Private Prosthodontics Practitioner, Pasadena, California

*Correspondence to: Dr Thomas Rose, 17900 Brookhurst St, Suite C, Fountain valley, CA 92708-5161

Email: tomrosedds@aol.com
}

\section{Refereed Paper}

๑ British Dental Journal 2006; 201: 753-764

DOI: $10.1038 / \mathrm{sj}$. bdj.4814349 tions should be made prior to the extraction. Too often teeth are extracted needlessly only to result in restorations which exhibit disproportionate relationships to the adjacent teeth (Figs 1-2). If bone deficiencies are present, orthodontic eruption of the tooth prior to extraction can help to increase the amount of hard and soft tissue in the future implant site. Orthodontic extrusion allows the restorative dentist to create a more harmonious gingival level and aesthetically provide the patient with a restoration that mimics the contra-lateral tooth. Extrusion is performed at a rate of $1 \mathrm{~mm}$ per week and a stabilisation period of one month for each millimeter extruded ${ }^{1}$ (Figs 3-10). Extrusion is the most predictable method of vertical bone development. It is likely that patients requiring dental implants to replace multiple missing teeth will require horizontal bone augmentation in addition to extrusion. The extrusion allows the clinician to develop the peri-implant site vertically so as to give the patient aesthetically proportionate restorations (Figs 11-16).

\section{Orthodontics to create space for implant restorations}

Orthodontic therapy is also invaluable in creating space for implant placement. When teeth are lost, opposing teeth often over-erupt or drift into the edentulous space, compromising space for the restoration. The restorative dentist needs to communicate with the orthodontist 
Fig. 1 Inadequate implant site development resulting in disproportionate restorations replacing upper left central and lateral incisors.

Fig. 2 Inadequate implant site development resulting in disproportionate implant supported restoration on upper left lateral incisor.

Fig. 3 Pre-operative clinical situation showing periodontally failing upper right lateral incisor.

Fig. 4 Periapical radiograph showing endodontically treated upper right central and lateral incisors.
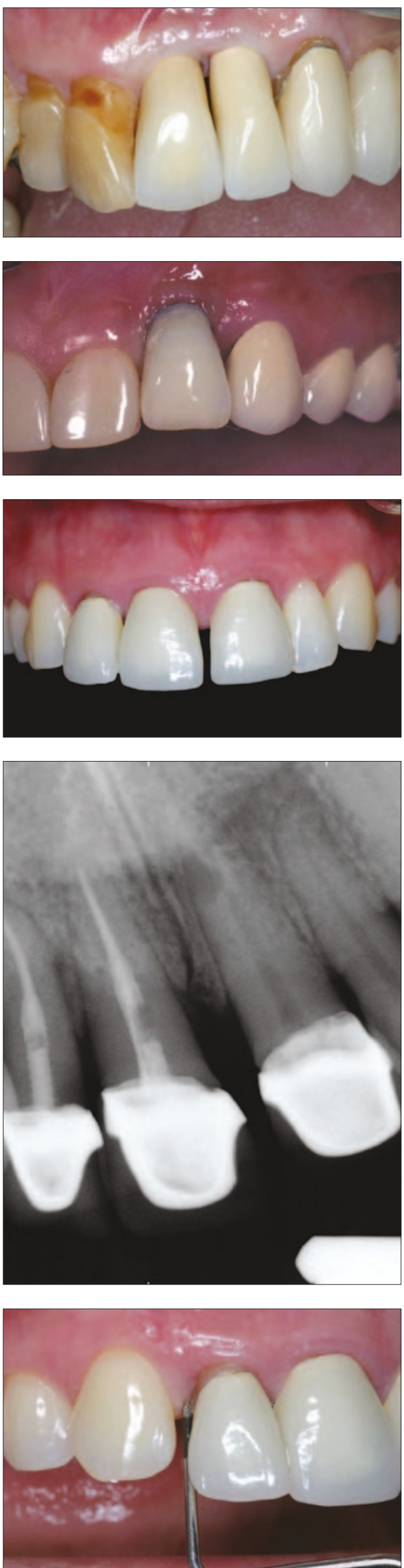

with regards to mesiodistal space required for an anatomically contoured restoration. Contra-lateral teeth can be used as a reference to communicate spatial requirements. A diagnostic wax up can also aid as a visual tool for the orthodontist so that appropriate space is created. Nothing is more disappointing for the patient than to go through lengthy orthodontic therapy only to have the restorative dentist to come to the conclusion that inadequate space exists. Orthodontic bands should not be removed until the restorative space has been evaluated and approved (Figs 17-19).

When lateral incisors are congenitally missing it is not uncommon to find that the adjacent roots drift into the space, making placement of implants difficult (Figs 20-22). Often orthodontic therapy is required to create space for implant placement and also for the prosthetic restoration. One difficulty that arises is communication of space required to the orthodontist.

An aesthetic relationship exists between the size of the central and lateral incisors. This ratio has been termed the Golden Proportion - it is not absolutely correct in all cases but serves as a very good guideline (Fig. 23). Ideally the maxillary lateral incisor should be two thirds the width of the central incisor. Most central incisors are $8-10 \mathrm{~mm}$ wide. If the central incisor is 8 $\mathrm{mm}$ in width then the lateral should be 5.5 , if the central is $9 \mathrm{~mm}$ then the lateral should be $6 \mathrm{~mm}$, if it is $10 \mathrm{~mm}$ then the lateral should be $6.7 \mathrm{~mm}$. The range of most lateral incisors varies from 5.5-6.7 $\mathrm{mm}$. In some situations the orthodontist may create less than ideal width for the lateral incisor. If space is not available the orthodontist should consider removing enamel interproximally from the central incisors and the canines to provide additional width for the central incisor crown.

The traditional width of an implant is 3.75 $\mathrm{mm}$. The platform of the implant is $4.00 \mathrm{~mm}$. This measurement is important because the platform must fit interproximally between the central incisor and the canine at the crest of the alveolar ridge. If the orthodontist has created 5.5 $\mathrm{mm}$ of space and the platform of the implant is $4 \mathrm{~mm}$ wide, then $0.75 \mathrm{~mm}$ of space will remain between the implant and the adjacent central incisor and canine teeth. There should be at least $1 \mathrm{~mm}$ of space between the implant and adjacent teeth This space allows adequate healing and the development of the papilla. If the space is narrow, consideration must be given to use of a smaller diameter implant. The orthodontist can benefit from a diagnostic wax up; this will allow visualisation of the end result and allow optimum outcomes for the patient (Figs 24-26).

\section{Implants for orthodontic anchorage}

For every force applied there is an equal and opposite force; this is one of Newton's laws of physics and much of the basis for the mechanics of tooth movement in orthodontics. Traditionally, the dentition with various intraoral and/or extra-oral appliances is used to 
create anchorage for alignment and retraction of teeth. During orthodontic treatment, the planned movement of one tooth or group of teeth causes reciprocal movement of the teeth used for anchorage. Gauging force dynamics is difficult as unwanted tooth movements often occur. These unwanted movements must be compensated for. Interdisciplinary therapy in the adult patient can be very challenging, particularly in situations where the patient exhibits a severe attachment loss from missing teeth or severe periodontal disease. This attachment loss adds to the difficulty of estimating anchorage and stability treatment becomes exponentially more difficult.

Any practitioner who has attempted unilateral movement of a third molar into the second molar position has observed the entire dental arch move toward the affected side. To state that anchorage is difficult to control and final tooth position is unpredictable would not be inaccurate.

Implants as an adjunct to orthodontic therapy are quickly gaining acceptance. They provide the qualities of an ideal orthodontic anchor: 1) patient compliance unnecessary; 2) absolute anchorage as there is no periodontal ligament; 3) easily used under a variety of treatment modalities; 4) easily placed; and 5) removable, if necessary.

\section{TYPES OF IMPLANT ANCHORS}

Definitive implants - Osseointegrated root form implants have shown remarkable success rates both in full and partial edentulism. Their application in orthodontics has become state of the art. Osseointegrated oral implants provide the necessary anchorage for the orthodontic tooth alignment and re-establishment of proper occlusion through tooth movement and prosthetic restorations.

Special care must be taken with the use of definitive implants in growing patients. Similar to ankylosed teeth, osseointegrated implants will not move with growth and the implant will be in infraocclusion when compared with the neighbouring erupting teeth. Endosseous implant insertion before the end of the growth of the jaws is therefore looked upon with reluctance. Hand wrist radiographs give an indication of the overall growth of a patient. Whether growth is expected and superimposition of two lateral cephalometric radiographs taken at an interval of six months will give more detail on growth of the jaws.

The implants range in diameter from 3.5-8.0 $\mathrm{mm}$, and 11-21 $\mathrm{mm}$ in length (Fig. 27A). They require approximately $8.0 \mathrm{~mm}$ of edentulous space for placement, and require three to six months for osseous integration before application of force. The implant will need an abutment with a provisional or definitive restoration for attachment of orthodontic appliances. Detailed treatment planning is required to obtain a diagnostic setup in order to determine location of the implant(s); Smalley reported this technique in 1995. 2,3 Typically these implants will

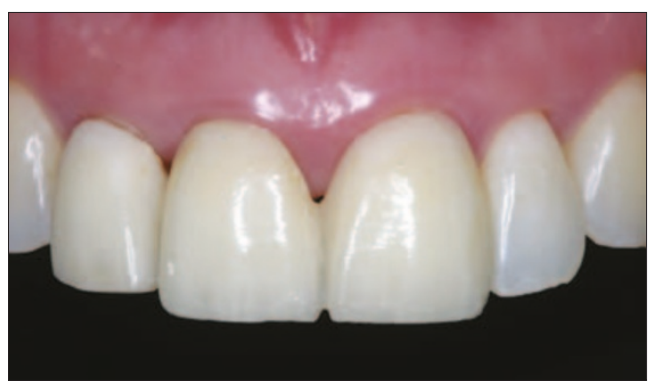

Fig. 6 Provisional restoration on upper right lateral and central incisors.

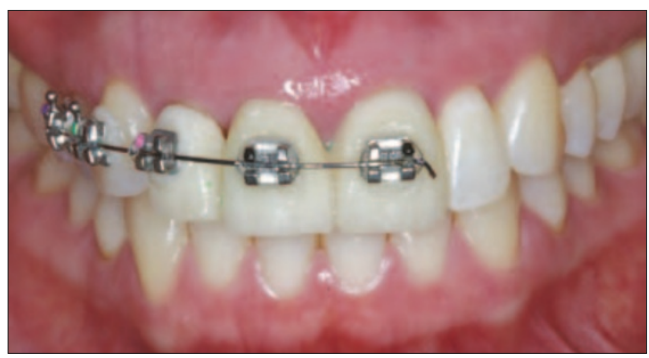

Fig. 7 Orthodontic therapy initiated to extrude the upper right lateral incisor for peri-implant site development.
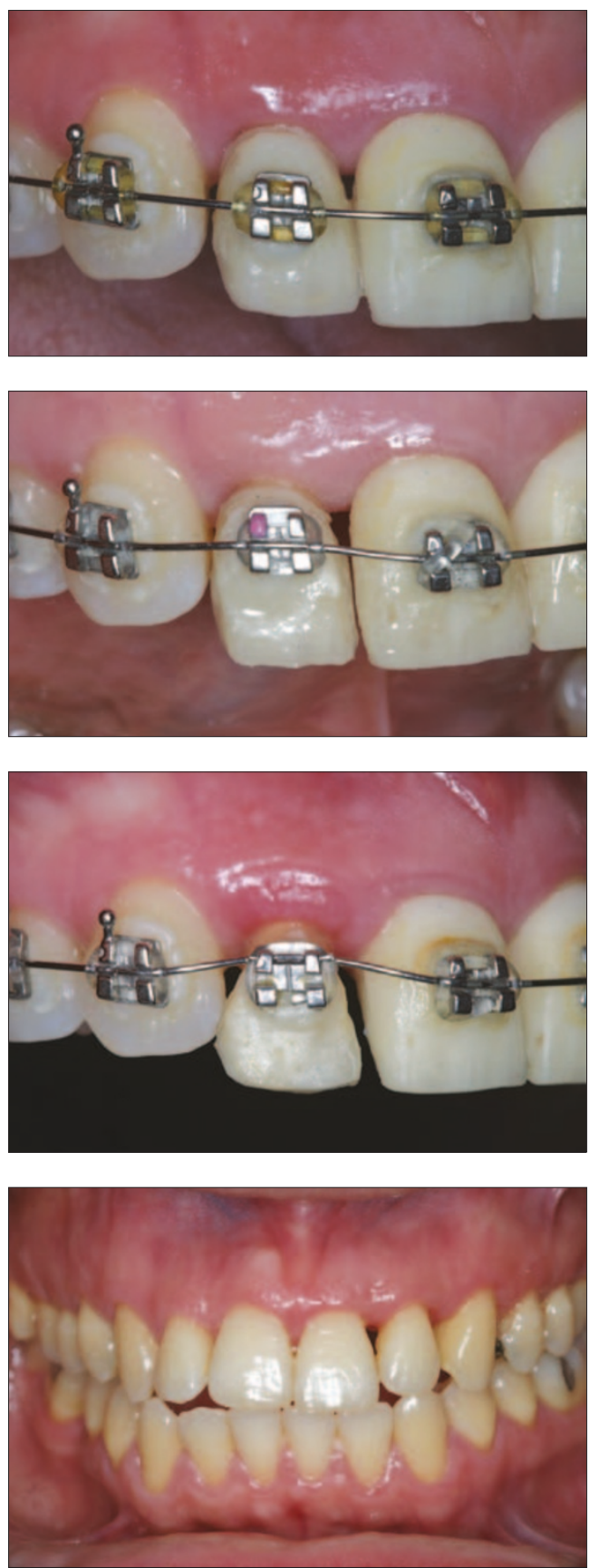

Fig. 9 Extrusion at two months.

Fig. 10 Extrusion at three months.

Fig. 8 Extrusion at one month.

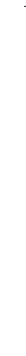

Fig. 11 Clinical preoperative situation, both lateral and central incisors are failing periodontally. 


\section{PRACTICE}

Fig. 12 (left) Orthodontic therapy initiated following periodontal surgery to extrude lateral and central incisors for peri-implant site development.

Fig. 13 (right) Extrusion at one month.

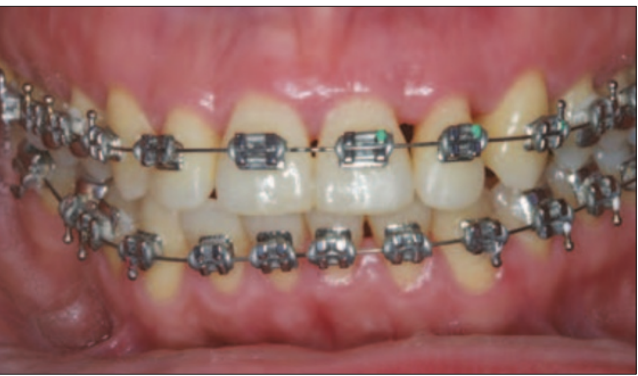

Fig. 14 (left) Extrusion at two months.

Fig. 15 (right) Extrusion at three months.

Fig. 16 (left) Clinical situation following stabilisation. There is a likelihood that autogenous bone grafting will be required to develop horizontal width.

Fig. 17 (right) Occlusal view demonstrating inadequate restorative space for lower right second premolar.

Fig. 18 (left) Orthodontic therapy initiated to open space for implant placement and restoration.

Fig. 19 (right) Implant restoration replacing lower right second premolar.

ig. 20 (left) Pre-operative radiograph of congenitally missing lateral incisors. Radiographs show tipping of adjacent roots compromising space required for implant placement.

Fig. 21 (right) Congenitally missing upper right lateral incisor.

Fig. 22 (left) Congenitally missing upper left lateral incisor.

Fig. 23 (right) Golden proportion gives the clinician some average guidelines to communicate with the orthodontist as to prosthetic space required.
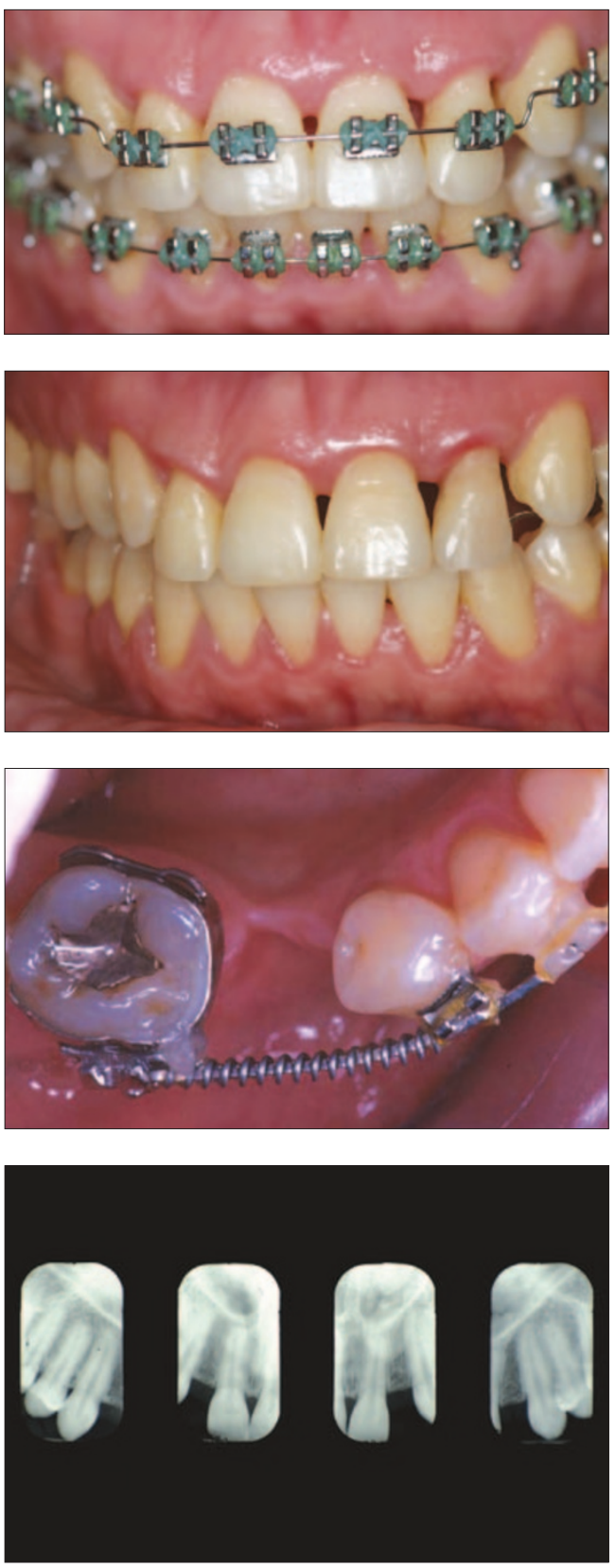
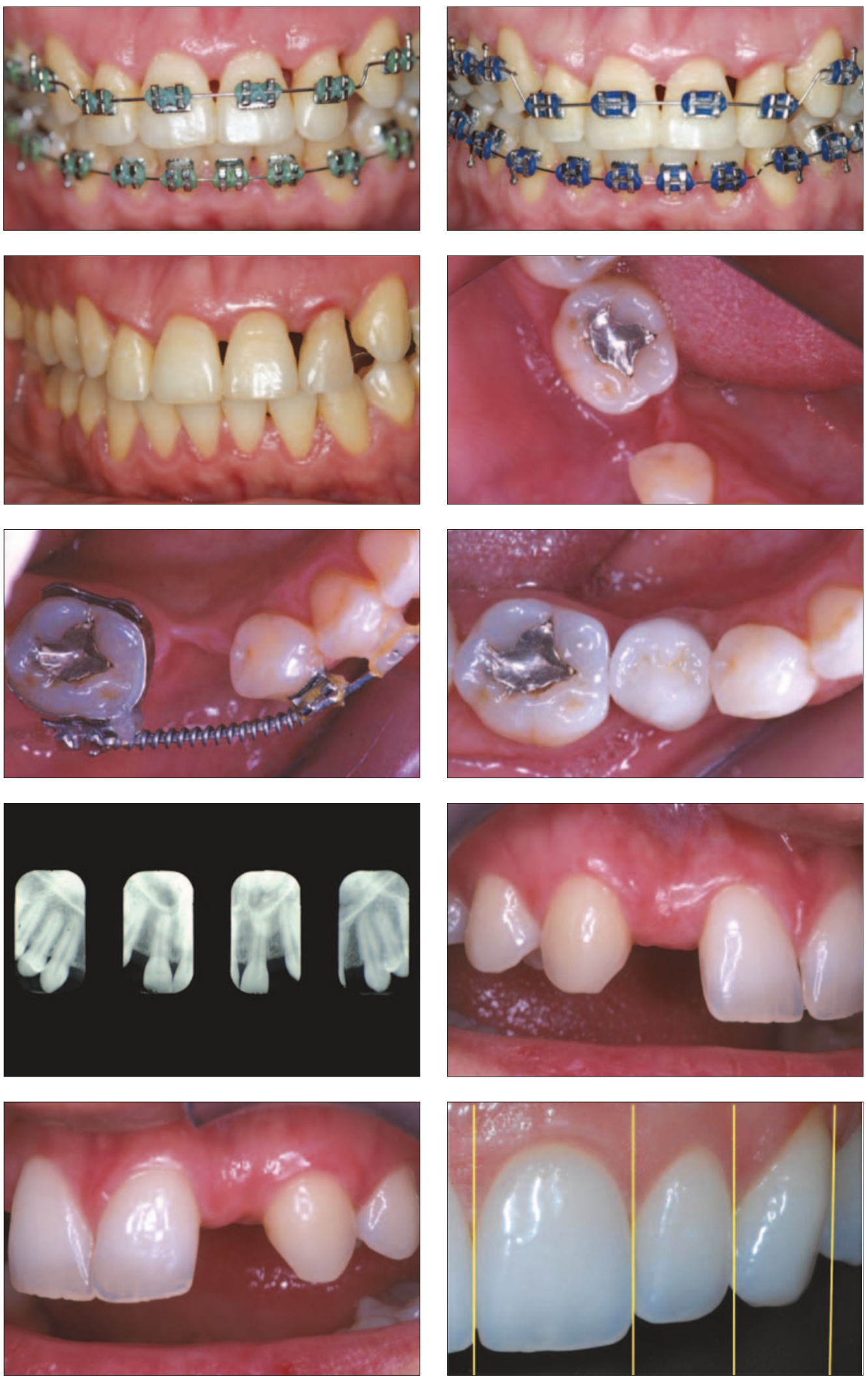

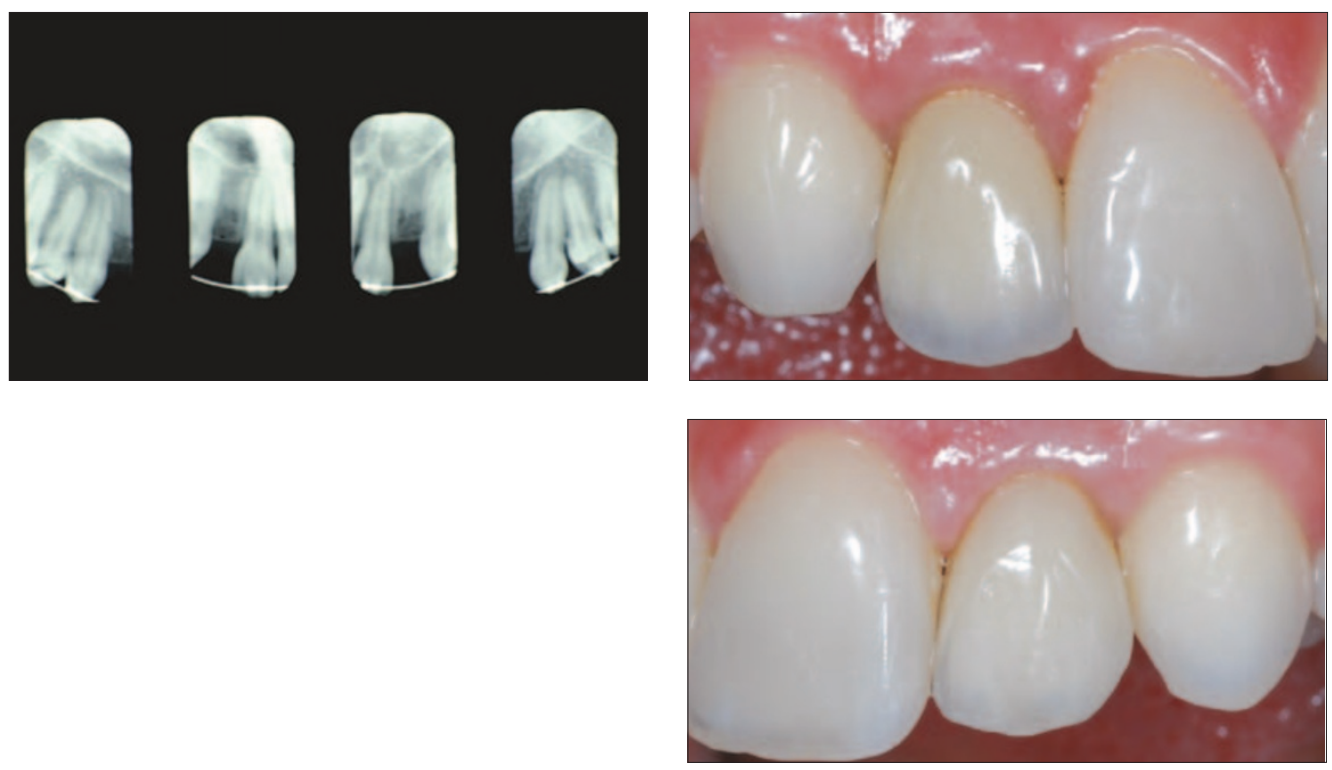

An ortho implant - (Straumann's Ortho support restorations or part of the reconstruction post-orthodontic movement. Therefore the implants are placed in relation to the ultimate positions of teeth and not the pre-treatment positions of teeth. Smalley described a method to relate implant positions to future tooth positions using precise diagnostic set ups and indices. This is the most difficult type of treatment incorporating implants, as once implants are placed, orthodontic therapy proceeds based on the position of the integrated implants and any revisions to the treatment plan may not be possible. Implants placed incorrectly will compromise the outcome of the treatment.

Disadvantages of root formed implants include:

1. Cost

2. Loading must be delayed for healing and osseointegration

3. Placement limited to edentulous and retromolar areas

4. Non restorable implants must be surgically removed after orthodontic use

5. Use in a growing patient may result in submergence of the implant.

Provisional implants - are typically 2.8-3.5 $\mathrm{mm}$ in diameter, and approximately $14 \mathrm{~mm}$ in length (Fig. 27B). They require $4 \mathrm{~mm}$ of edentulous space for placement. Immediate provisional implants are usually fabricated of a titanium alloy, and differ from definitive implants in two ways: 1) osseointegration is not expected, and 2) they are susceptible to fatigue fracture and mobility with long-term use. Therefore, replacement during orthodontic treatment should be expected. These implants depend on primary mechanical stability to provide orthodontic anchorage. A provisional restoration can be formed onto the implant to attach a bracket or appliances can be directly wired to the implant. They are appropriate for opening space for a full size implant such as the maxillary lateral incisor region. Orthodontic forces may be applied to the provisional implant immediately.
System) (Fig. 27C) requires 6-8 $\mathrm{mm}$ of space for placement. An abutment with a provisional crown, or fabrication of an appliance attached to the implant, is needed to apply orthodontic forces, and three months of osseous integration is recommended, prior to use.

Mini-implant - the mini-implant was introduced by Kanomi in $1997^{4}$ (Fig. 27D). He showed in a case report that titanium mini-implants were used to intrude mandibular incisors to correct a deep bite.

The mini-implant is basically a bone screw similar to the type used to fix bone plates for plastic reconstruction. It requires a two stage surgical procedure with an unloaded period for healing.

The mini-implant is $1.2 \mathrm{~mm}$ in diameter and $6 \mathrm{~mm}$ long, making it much more useful in orthodontic applications. Kanomi has extrapolated that as well as incisor intrusion, the miniimplants could be used for horizontal traction if placed on the alveolar ridge. The screw is small enough to be inserted between the mesial and distal roots of a molar for molar intrusion. Kanomi also points to the possible use of the implant in distraction osteogenesis, with the implant placed intra-orally instead of extraorally.

Ortho miniscrews and microscrews are usually $1.5-2.0 \mathrm{~mm}$ in diameter, and 5-8 $\mathrm{mm}$ in length. 5,6 They require minimal instrumentation for placement, since most are self-tapping screws. After two weeks, the anchor screws can tolerate orthodontic forces. ${ }^{19}$

Mini-plate - the mini-plate was developed and introduced by Umemori et al. in $1999,{ }^{7}$ as a skeletal anchorage system employing titanium mini-plates temporarily implanted in the maxilla or mandible with bone screws as an immobile intra-oral anchorage source. It is particularly used for intrusion of molars to correct the skeletal open bite. In the cases presented, a significant amount of molar intrusion was achieved, and this was associated with minimum
Fig. 24 (left) Post-orthodontic radiograph illustrating adequate space for placement of implants in lateral incisor region.

Fig. 25 (right) Final implant restoration of upper right lateral incisor.

Fig. 26 Final implant restoration of upper left lateral incisor. 


\section{PRACTICE}

Fig. 27A-D - Types of Implants: A) definitive implant with abutment or provisional restoration (Straumann Dental, Switzerland); B) provisional implant (Nobel Biocare, Sweden); C) ortho system implant (Straumann Dental); D) ortho miniscrew (OsteoMed, Addison, Texas).

Fig. 28A-G Pretreatment extraoral and intraoral photographs of patient $(\mathrm{JN})$.
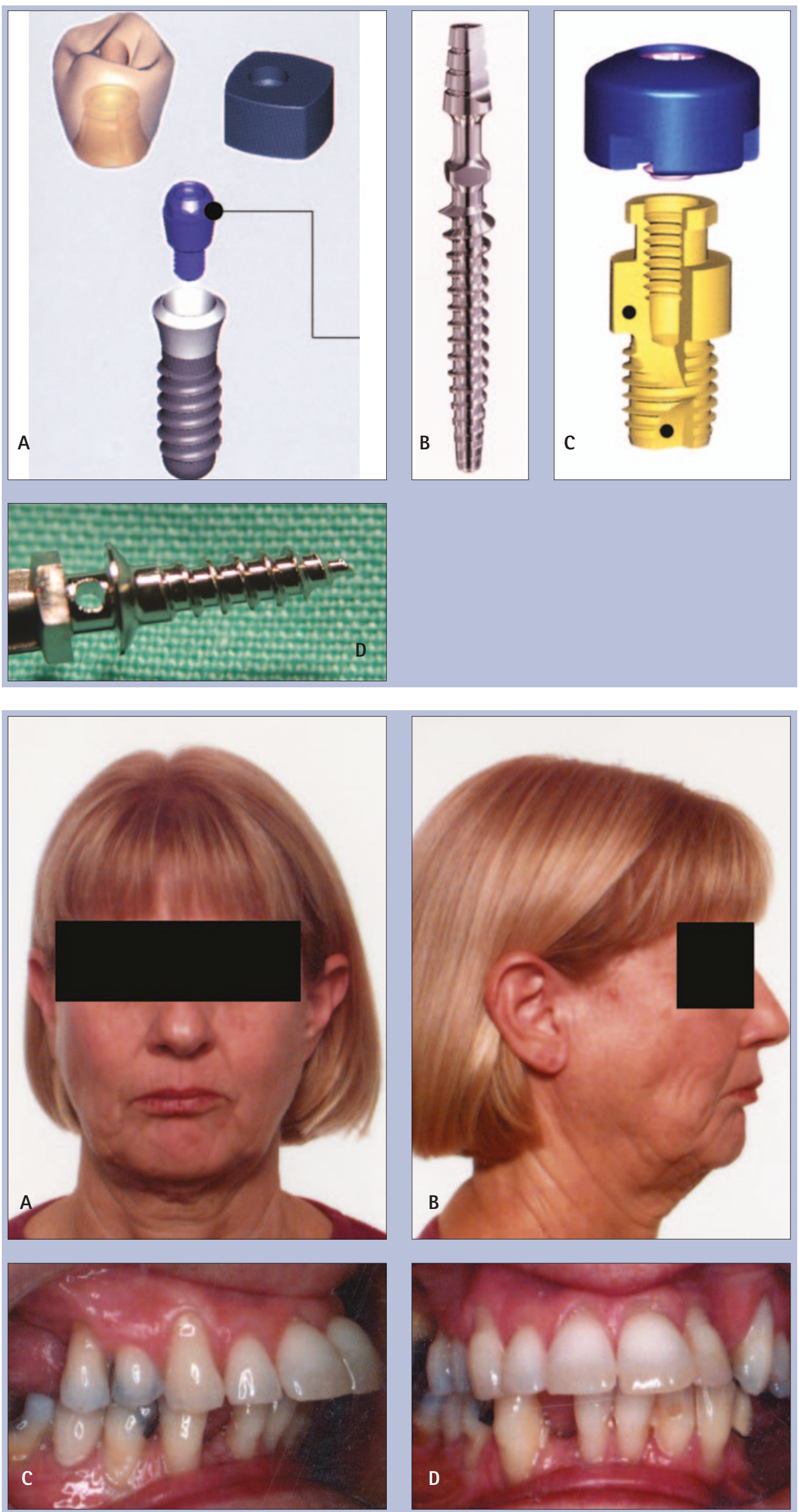

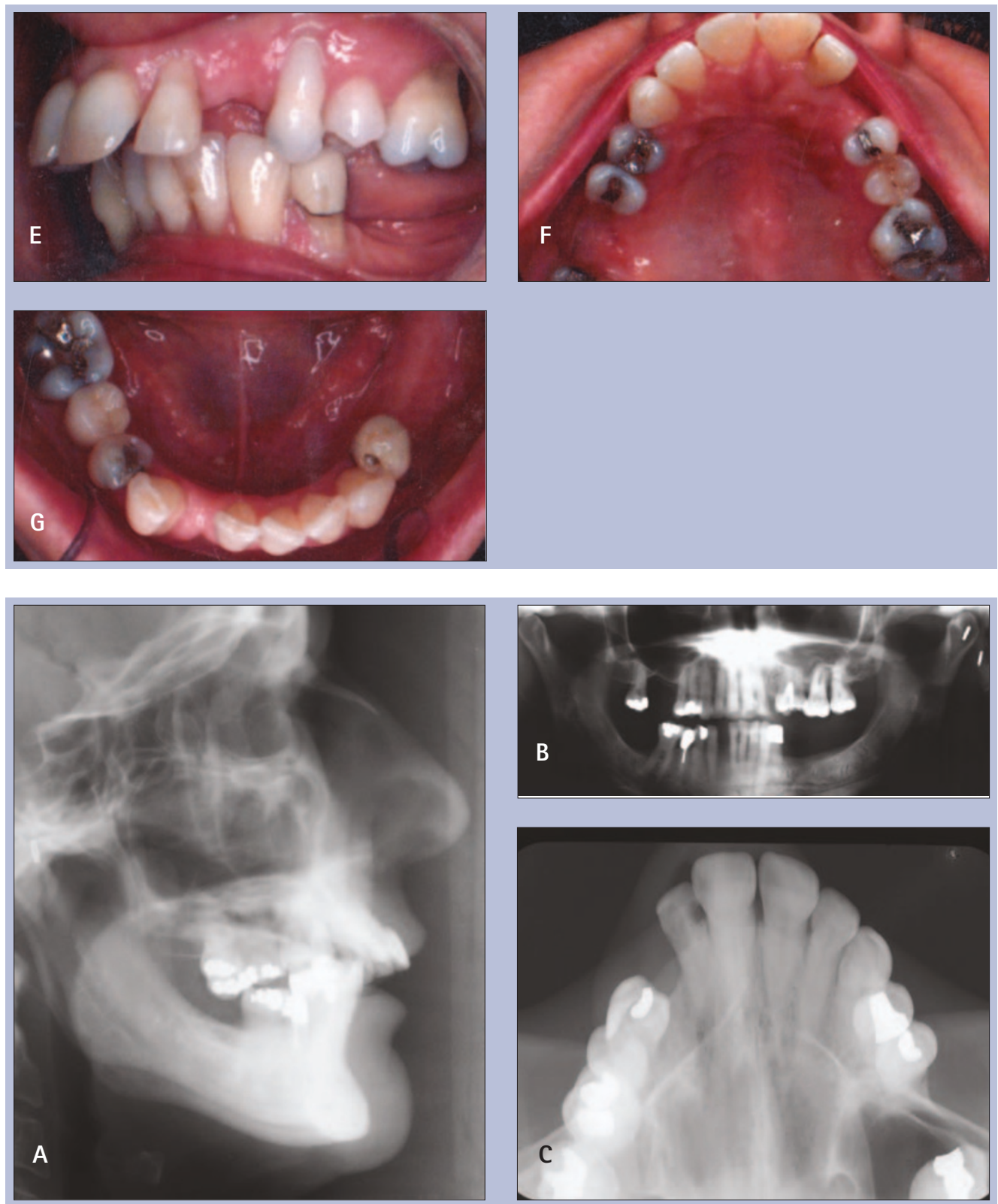

Fig. 29A-E Pretreatment

cephalometric, panoramic, occlusal, and periapical views.
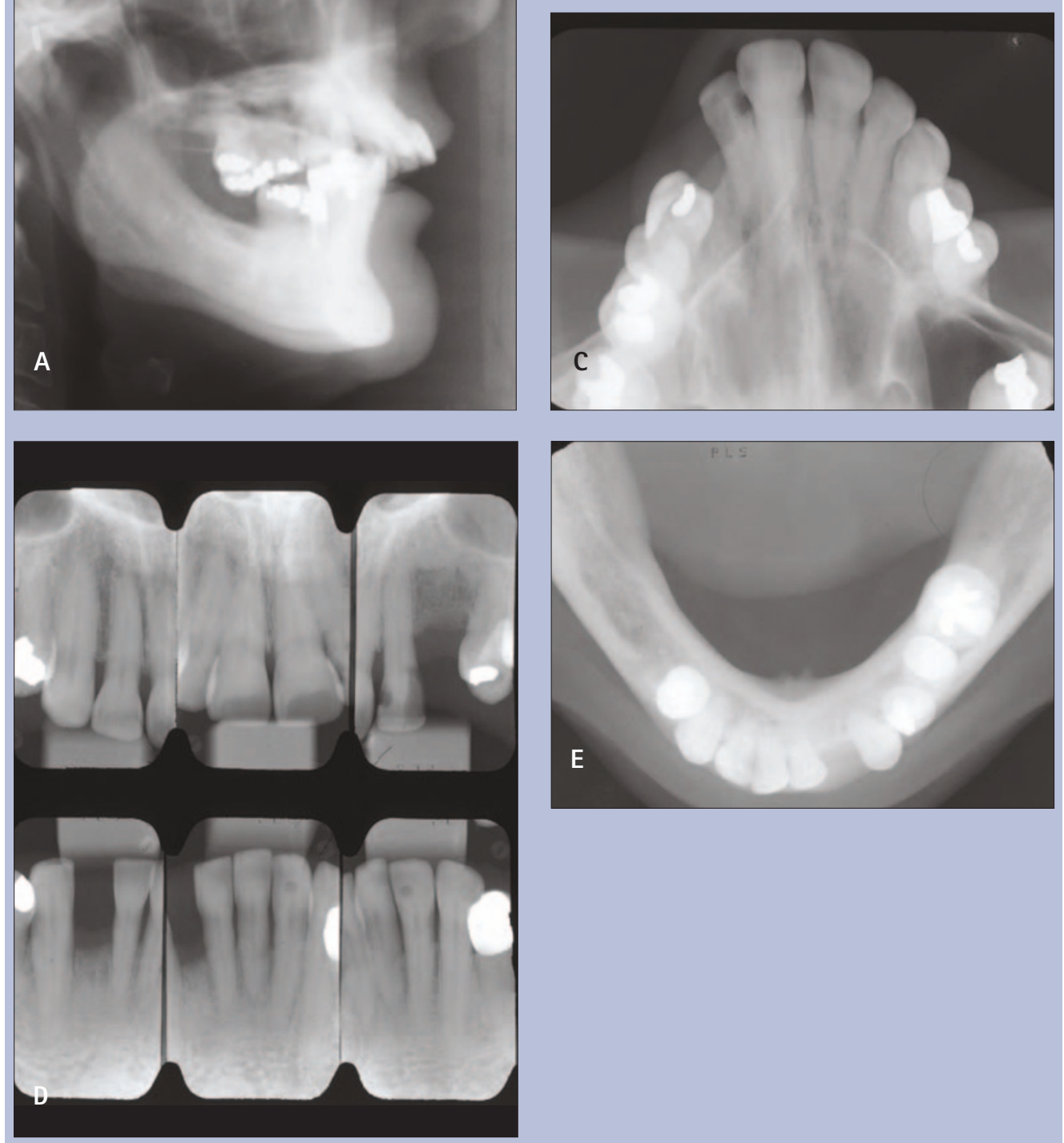


\section{PRACTICE}

Fig. 30A-F Diagnostic mounted cast and precision setup. Skeletal asymmetry is accurately demonstrated on the frontal view, resulting in a cant of the occlusal plane.
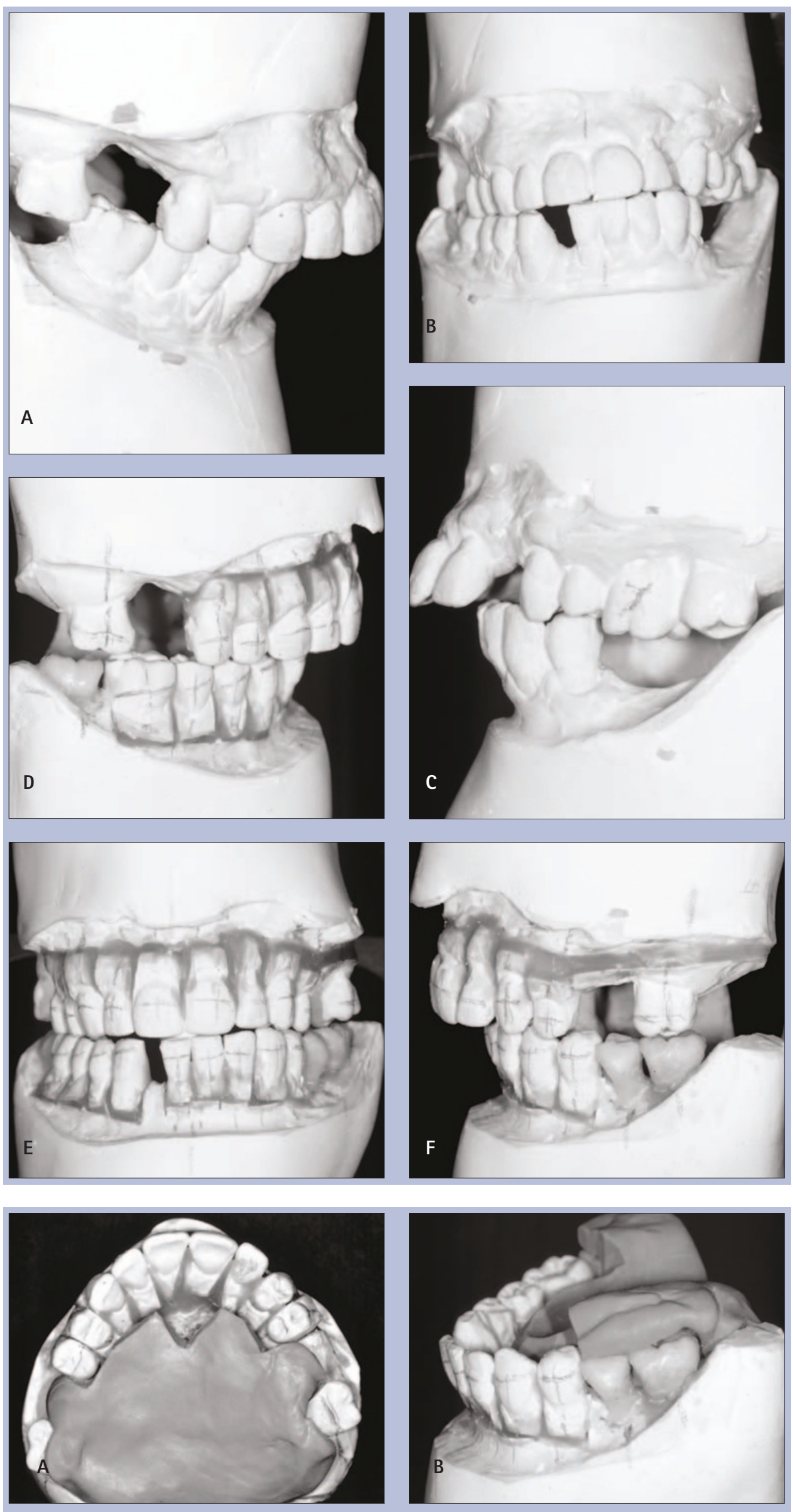

Fig. 31A-B Vinyl polysiloxane impression of the lingual surfaces and pontics. 

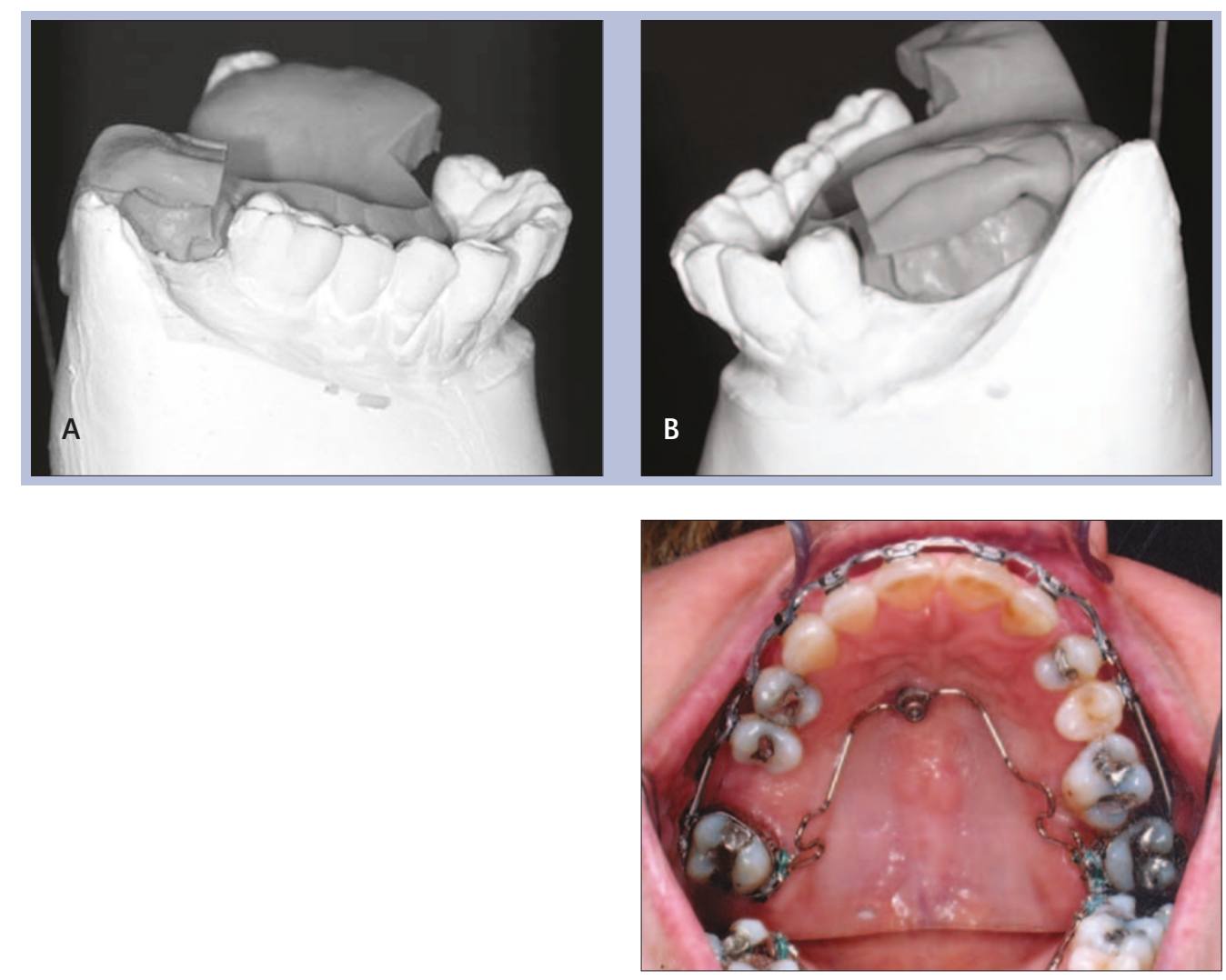

Fig. 33 Palatal implant and transpalatal archwire (0.036 inches) for posterior anchorage.

Fig. 32A-B Transfer silicone matrices to duplicate initial casts for proper positioning of implants. extrusion of the lower incisors and counterclockwise rotation of the occlusal plane.

\section{Background/historical review}

Vitallium screws were used by Gainsforth and Higley, as early as 1945, to apply orthodontic forces in dogs, although unsuccessfully. ${ }^{8}$ In 1983 Creekmore described using a metal screw to intrude anterior teeth, and the possibility of skeletal anchorage. ${ }^{9}$ Roberts et al. used springs to apply force to titanium screws in rabbits in 1984, and concluded the implants provided adequate anchorage for orthodontic force levels. ${ }^{7}$ Several investigators have reported the use of osseointegrated implants for uprighting molars to minimise adverse sequelae, such as extrusion. ${ }^{10-12}$ In 1997 Kanomi placed mini-bone screws between the root apices of the mandibular incisors to intrude the lower anteriors. ${ }^{4}$ More recently, Park, Lee, and an increasing number of investigators have discussed the use of microimplants for skeletal anchorage. , $^{5,13-20}$

\section{CASE REPORT}

A 56-year-old female patient (JN) presented with a severe Class II division 1 mutilated malocclusion with multiple missing teeth (Figs 28A-G). Her chief concerns were replacement of the missing teeth and correction of her overjet and dental protrusion. She reported a history of previous orthodontic treatment for four years from nine to 13 years of age. The patient indicated that her previous orthodontic treatment left her with residual overjet, but all inter-dental spaces were closed when her braces were removed. Facially, the patient had a convex profile with eversion of the lower lip, acute nasal-labial angle, and moderate retrusion of the chin. She exhibited a Class II skeletal pattern, secondary to a combined maxillary protrusion and mandibular hypoplasia, with the mandible deviated to the patient's right side (Figs 29A-E). Cephalometric analysis reflected an ANB difference of 6.5 degrees, a Wits measurement of 7.5 $\mathrm{mm}$, an FMA of 28.5 degrees, and A-P inclination of the maxillary and mandibular incisors at 116.5 degrees. Dentally, the patient exhibited 50\% horizontal bone loss in both dental arches, moderate to severe tissue recession, flaring of the maxillary anterior teeth with inter-dental spacing, and super-eruption of the maxillary molars due to lack of opposing dentition. Tooth number 14(27) exhibited $7 \mathrm{~mm}$ periodontal pockets with a very guarded prognosis.

\section{Treatment recommendations}

A comprehensive treatment approach involving periodontal therapy, orthodontics, prosthetics, and orthognathic surgery was recommended, and accepted by the patient. Periodontal therapy was accomplished prior to initiating orthodontic treatment to avoid exacerbating the existing bone loss and tissue recession. Tissue grafts were placed on the facial of teeth numbers 6(13), 10(22), 12(24), 21(33), and 22(33) to 28(44). Due to the lack of anchorage support and existing bone loss, implants would be required for orthodontic therapy. To assure proper placement of the implants, diagnostic mounted models were secured, duplicated, and a precision setup was accomplished, based on the protocol described by Smalley ${ }^{2,3}$ (Figs 30A-F). It is imperative the orthodontist accomplish the precision setup, since $s /$ he will be determining the final position 


\section{PRACTICE}

Fig. 34A-E Progress records taken 10 months after initiating retraction. Soft

tissue migrated occlusally, and bone

level has been maintained.
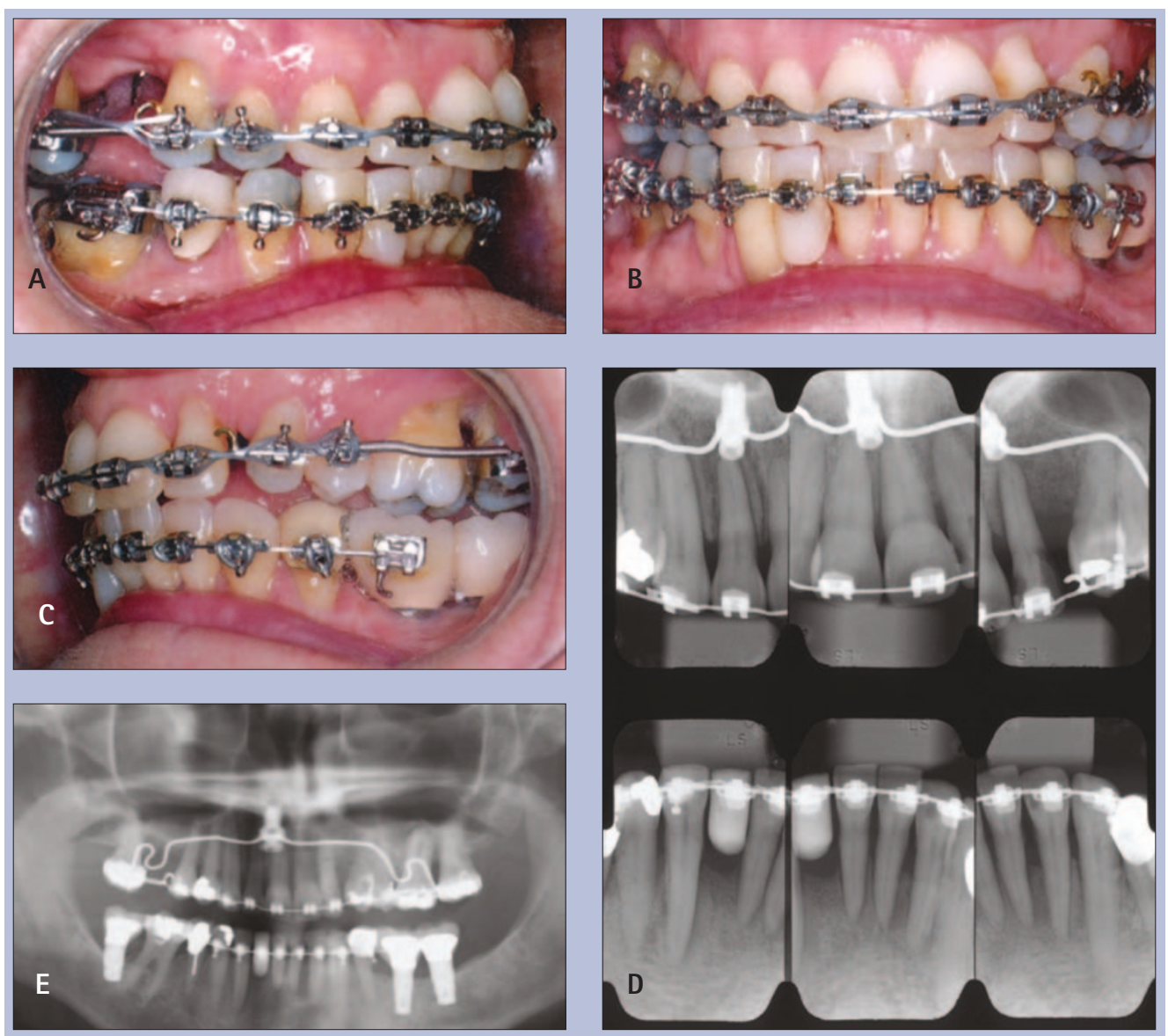

Fig. 35A-G Final extraoral and intraoral photographs of patient (JN).
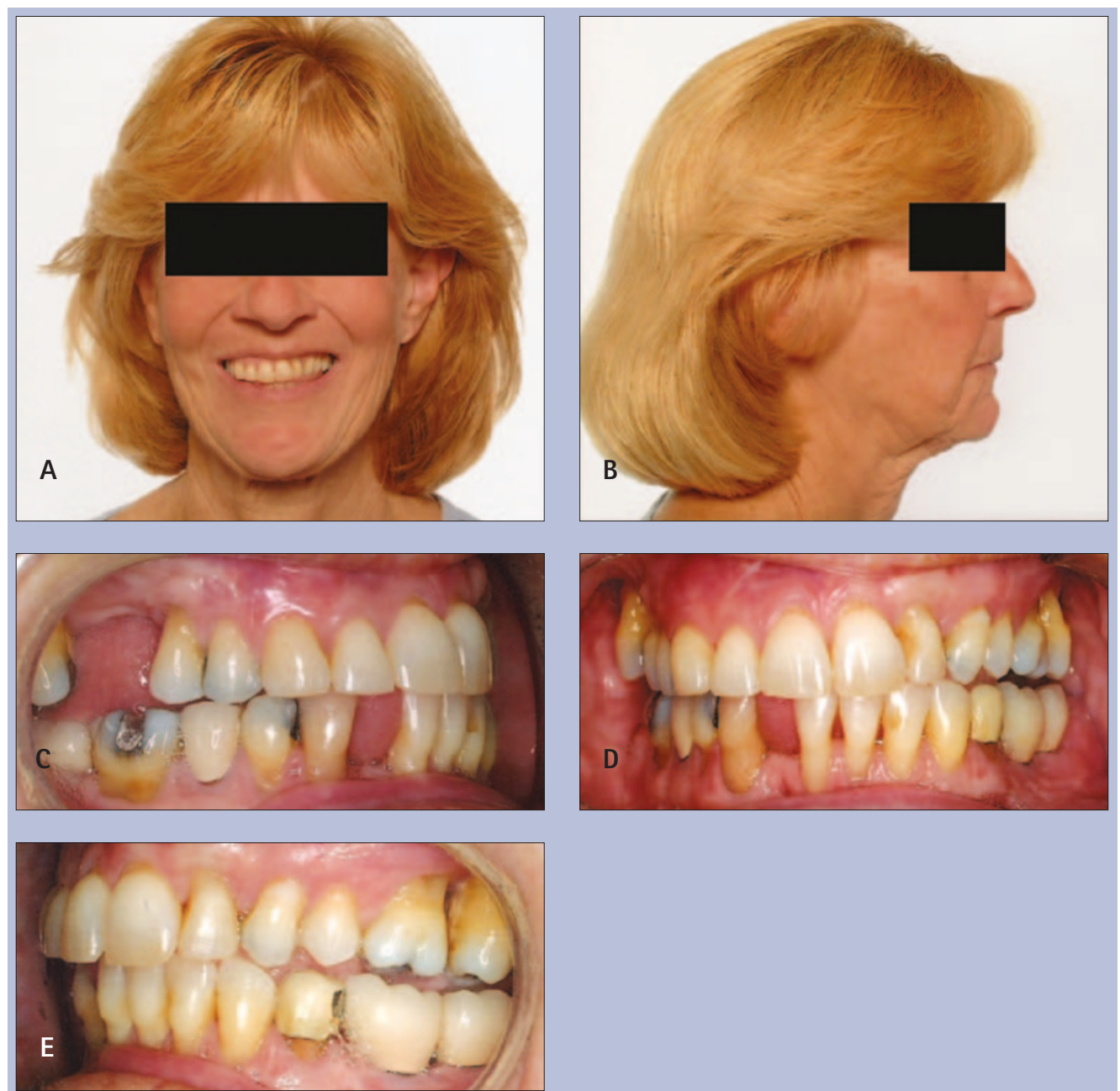


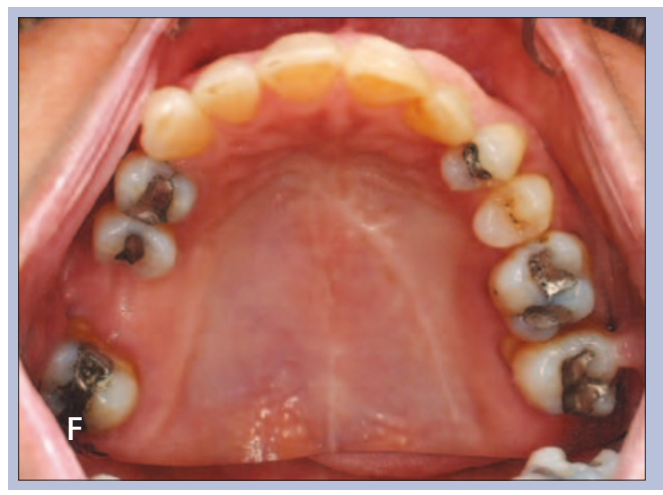

of the dentition. Transferring the information to the pretreatment cast and fabricating a surgical stint permits accurate placement of the implants (Figs 31A-B, 32A-B. Provisional fixed prosthetic restorations were placed on the mandibular implants for placement of orthodontic brackets. Due to super-eruption of the maxillary molars, the provisional crowns were deliberately fabricated with reduced clinical height to avoid occlusal interference during preliminary arch alignment. In the maxillary arch a provisional orthodontic anchor was placed in the anterior palate (Fig. 33) to reduce stress on the maxillary posterior teeth during retraction of the anteriors. Maxillary left first molar (number 14 [26]) was removed for periodontal considerations, prior to commencing orthodontic therapy.

\section{Treatment progress}

A transpalatal archwire (0.036) was adapted to rest against the palatal implant, which prevented the molars from moving during retraction of the anterior teeth, and permitted slight intrusion of the maxillary molars. The palatal implant was permitted to integrate for three months prior to application of force. Ten months after initiating retraction, preliminary arch alignment was completed with the anteriors uprighted over their respective apical bases (Figs 34A-E). The patient then had orthognathic surgery involving: 1) maxillary osteotomy to impact the posterior to level the occlusal plane and widen the arch $5 \mathrm{~mm}$; 2) mandibular advancement osteotomy with rotation to the patient's left side to correct the skeletal asymmetry to achieve a class I dental and skeletal relationship; and 3) an augmentation genioplasty to improve the patient's chin projection and facial muscle balance. The genioplasty was later modified to reduce the projection of the chin at the patient's request. After stabilising and detailing the occlusion, her appliances were removed and retainers were placed (Figs 35A-G, 36A-E).

\section{Discussion}

The retraction and uprighting of the anterior teeth over their apical bases resulted in the gingival tissue migrating occlusally, with little or no loss of osseous support for the teeth. This would have been difficult, if not impossible, to achieve without skeletal anchorage.

Prior to treatment, the patient had received

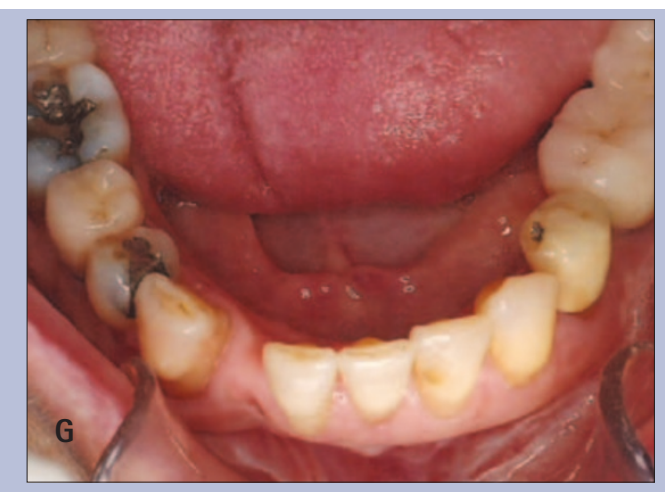

opinions from two other orthodontists that treatment would result in loss of her teeth. Given the patient's pretreatment periodontal condition, bone loss, and multiple missing teeth, conventional orthodontics alone would have been futile. A Class I occlusion with normal overbite and overjet were achieved. The bilateral posterior openbite was deliberately created. Attempting to extrude the maxillary molars orthodontically would have resulted in a reverse smile line, and undone the aesthetic improvement achieved through leveling the maxillary arch surgically. The patient is now ready for her prosthetic phase of care. This will include replacing the provisional crowns on the implants in the area of teeth numbers 18, 19, and 31. Additional implants and/or fixed prosthetic restorations will be needed to replace teeth numbers 3 and 26 , and a new crown on tooth number 21 . The patient's profile and symmetry were improved with the aid of orthognathic surgery.

This case demonstrates the need for a coordinated treatment approach in complex and mutilated malocclusion, and that implants and skeletal anchorage can provide reliable support for orthodontic treatment.

\section{SUMMARY}

Osseointegrated implants have shown remarkable success both in full and partial edentulism, their application in orthodontics has become state of the art. The use of implants for orthodontic anchorage can produce superior preprosthetic tooth movements for partially edentulous patients. However their use requires that a prosthesis be fabricated to optimally direct orthodontic forces between the implants and the teeth to be moved.

Interdisciplinary planning can contribute to a successful use of the implants. A three dimensional planning of the implant location site is necessary in order not to compromise orthodontic movement or subsequent prosthetic treatment.

1. Salama H, Salama M, Kelly J. The orthodontic-periodontal connection in implant site development. Pract Periodont ics Aesthet Dent 1996; 8: 923-932.

2. Smalley W M. Implants for tooth movement. Determining implant location and orientation. J Esthet Dent 1995; 7: 62-72.

3. Smalley W M. Implants for tooth movement. A fabrication and placement technique for provisional restorations. J Esthet Dent 1995; 7: 150-154. 
Fig. 36A-D Final cephalometric, panoramic, occlusal, and periapical views.

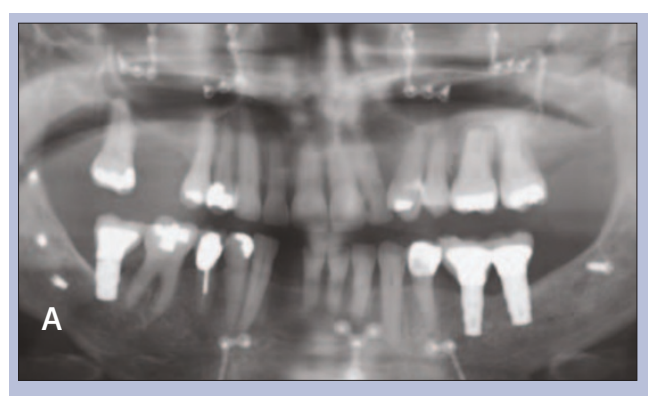

4. Kanomi R. Mini-implant for orthodontic anchorage. J Clin Orthod 1997; 31: 763-767.

5. Paik CH, Woo Y J, Kim J, Park JU. Use of miniscrews for intermaxillary fixation of lingual-orthodontic surgical patients. J Clin Orthod 2002; 36: 132-136.

6. Bae S M, Park H S, Kyung H M, Kwon O W, Sung J H. Clinical application of micro-implant anchorage. J Clin Orthod 2002; 36: 298-302.

7. Umemori M, Sugawara J, Mitani H, Nagasaka H, Kawamura H. Skeletal anchorage system for open-bite correction. Am J Orthod Dentofacial Orthop 1999; 115: 166-174.

8. Gainsforth B L, Higley L B. A study of orthodontic anchorage possibilities in basal bone. Am J Orthod Oral Surg 1945: 31: 406-417.

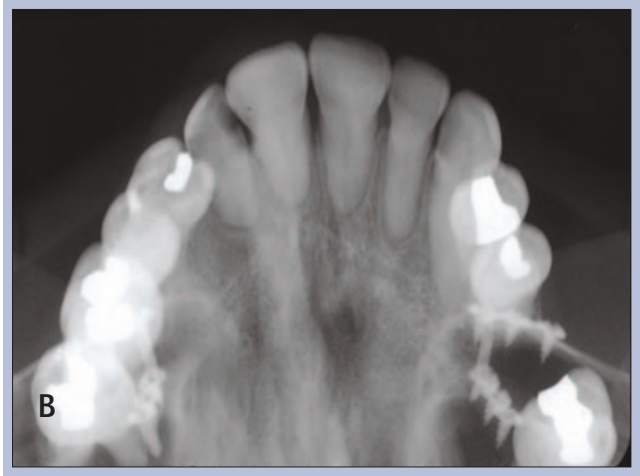

9. Creekmore T D, Eklund M K. The possibility of skeletal anchorage. J Clin Orthod 1983; 17: 266-269.

10. Roberts W E, Smith R K, Zilberman Y, Mozsary P G, Smith R S. Osseous adaptation to continuous loading of rigid endosseous implants. Am J Orthod 1984; 86: 95-111.

11. Park HS Kyung H M, Sung J H. A simple method of molar uprighting with micro-implant anchorage. J Clin Orthod 2002; 36: 592-596.

12. Park H S, Bae S M, Kyung H M, Sung J H. Micro-implant anchorage for treatment of skeletal class I bialveolar protrusion. J Clin Orthod 2001; 35: 417-422.

13. Park HS. The use of micro-implants as orthodontic anchorage. Seoul, Korea: Nare Publishing Co, 2001.

14. Deguchi T, Takano-Yamamoto T, Kanomi R, Hartsfield J K Jr, Roberts W E, Garreto L P. The use of small titanium screws for orthodontic anchorage. J Dent Res 2003: 82: 377-381.

15. Lee J S, Park HS, Kyung H M. Micro-implant anchorage for lingual treatment of a skeletal class II malocclusion. J Clin Orthod 2001; 35: 643-647.

16. Bae S M, Park H S, Kyung H M, Sung J H. Ultimate anchorage control. Texas Dent J 2002; 119: 580-591.

17. Fritz U, Ehmer A, Diedrich P. Clinical suitability of titanium microscrews for orthodontic anchorage-preliminary experiences. J Orofac Orthop 2004; 65: 410-418.

18. Park H S, Bae S M, Kyung H M, Sung J H. Simultaneous incisor retraction and distal molar movement with microimplant anchorage. World J Orthod 2004; 5: 164-171.

19. Park HS, Kwon T G, Sung J H. Nonextraction treatment with microscrew implants. Angle Orthod 2004; 74: 539-549.

20. Giancotti A Greco M, Mampieri G, Arcuri C. The use of titanium miniscrews for molar protraction extraction treatment. Prog Orthod 2004; 5: 236-247. 